\title{
Pigeons Encode Absolute Distance but Relational Direction From Landmarks and Walls
}

\author{
Emily R. Gray and Marcia L. Spetch \\ University of Alberta
}

\begin{abstract}
In recent studies, researchers have examined animals' use of absolute or relational distances in finding a hidden goal. When trained with an array of landmarks, most animals use the default strategy of searching at an absolute distance from 1 or more landmarks. In contrast, when trained in enclosures, animals often use the relationship among walls. In the present study, pigeons were trained to find the center of an array of landmarks or a set of short walls that did not block external cues. Expansion tests showed that both groups of pigeons primarily used an absolute distance strategy. However, on rotational tests, pigeons continued to search in the center of the array, suggesting that direction was learned in relation to array.
\end{abstract}

Keywords: spatial cognition, absolute distance, relational direction, landmark configurations

In recent studies, researchers have examined how animals use geometric information provided by the shape of an enclosure or an array of landmarks to locate a goal. Gallistel (1990) noted that "shape is largely defined by the distances between and along surfaces (or lines) and by the angles they form, that is, by the uniquely metric relations" (p. 172). Shape can be encoded in terms of absolute or relational information. Absolute encoding would entail learning specific metrics, such as a specific distance and/or direction from a landmark or wall to the goal. In contrast, relational encoding involves learning the location of the goal in relation to two or more features of the environment and may involve learning a rule such as "in the middle" or "equidistant."

Researchers have used two common approaches to studying how organisms use geometric information to orient. In one approach, researchers investigate reorientation in an environment that provides salient but ambiguous geometry (see Cheng \& Newcombe, 2005, for a review). For example, in a rectangular environment, any location in the rectangle has an identical rotational equivalent and, hence, rotational errors indicate use of geometry. Organisms can also use featural information (such as a differently colored wall or distinct landmarks), if available, to disambiguate the environment.

Cheng (1986) was the first to use a reorientation paradigm to investigate how rats encode the geometric and featural properties

Emily R. Gray and Marcia L. Spetch, Department of Psychology, University of Alberta, Edmonton, Alberta, Canada.

This research was supported by a Natural Sciences and Engineering Research Council of Canada research grant (Discovery Grant 38861) awarded to Marcia L. Spetch. Some of these data were presented at the 13th Annual International Conference on Comparative Cognition, Melbourne Beach, Florida, March 2006. We thank Alisha Brown, Danielle Ludeman, Scott McAnsh, Clara Tsang, and Alexandra Twyman for assistance in the research, and Bernd Kohler and Isaac Lank for technical assistance.

Correspondence concerning this article should be addressed to Emily R. Gray, Department of Psychology, BSP-217, University of Alberta, Edmonton, Alberta T6G 2E9, Canada. E-mail: egray@ualberta.ca of a rectangular test environment. He found that rats relied almost exclusively on geometry in working memory tasks, frequently making rotational errors even though featural information made the environment unambiguous. In reference memory tasks, rats eventually used featural information to locate the correct corner but continued to prefer geometric information. Cheng concluded that rats first process the overall shape of the environment and then "paste on" featural information to the metric frame.

Use of the geometric shape of a rectangular enclosure to reorient has since been observed in many other organisms, including human adults and children, monkeys, fish, and birds (see review by Cheng \& Newcombe, 2005). Although use of geometry appears to be general, some species such as chicks (Gallus gallus) and pigeons rely more on the featural properties of the environment when features and geometry are put in conflict (e.g., Kelly, Spetch, \& Heth, 1998; Vallortigara, Zanforlin, \& Pasti, 1990). In human children (Learmonth, Nadel, \& Newcombe, 2001), chicks (Vallortigara, Feruglio, \& Sovrano, 2005), and fish (Sovrano, Bisazza, \& Vallortigara, 2005), the absolute size of the rectangular environment influences the extent to which features are used. In pigeons, scale transformation tests have provided some evidence that geometric information is encoded at least partly in a relational fashion (Kelly \& Spetch, 2001).

A second common approach to investigation of the use of geometry is training an organism to find the goal in the center of an enclosure or an array of landmarks. In enclosed environments, animals seem to readily use the geometric relationships among walls to find a goal. For example, chicks trained to find food in the center of enclosed spaces of various geometric shapes divided their searches between (a) areas corresponding to the absolute learned distance from the walls and (b) an area in the center when tested in expanded enclosures (Tommasi \& Vallortigara, 2000; Tommasi, Vallortigara, \& Zanforlin, 1997). Similar results were found with pigeons (Gray, Spetch, Kelly, \& Nguyen, 2004).

Interestingly, use of relational metrics in enclosures stands in contrast to many landmark array studies. With landmark arrays, most species seem to rely on absolute distances unless specifically 
trained to use relational metrics. Spetch et al. (1997) trained pigeons to find food in the center of a square array of identical landmarks. When the array was expanded, the pigeons searched at the absolute learned distance from one or more of the landmarks. They did, however, appear to use the configuration of the array to identify the landmarks (e.g., to determine which was the top left landmark). Gerbils (Meriones unguiculatus) and monkeys (Macaca mulatta) also have shown little or no relational responding on landmark array expansion tests (see review by Spetch \& Kelly, 2006). The use of absolute metrics with landmark arrays appears to reflect a preferred strategy rather than an inability to encode landmarks relationally. Specifically, when trained with landmark arrays that maintain shape but vary in absolute metrics, Clark's nutcrackers (Nucifraga columbiana; Kamil \& Jones, 1997, 2000) and pigeons (Jones, Antoniadis, Shettleworth, \& Kamil, 2002; Spetch, Rust, Kamil, \& Jones, 2003) showed relational learning. Nevertheless, when trained with constant interlandmark distances, nutcrackers, similar to pigeons, appear to use absolute landmarkto-goal distances on expansion tests (D. M. Kelly, personal communication, May 29, 2003).

Thus, encoding strategies are flexible, and the preferred strategy seems to differ according to the task that the animal is performing. In landmark array tasks, the preferred strategy of most species is use of absolute metrics, whereas in enclosure tasks, several species readily attend to relational cues from walls. This could represent a difference in the way that animals encode information from discrete landmarks versus from extended surfaces. Alternatively, the strategy difference could reflect other differences between the landmark and enclosure tasks, such as the availability of external cues. Orientation cues external to the experimental array are available in the landmark tasks, but these cues typically are blocked in the enclosures. These external cues may allow the landmarks to serve as purely local cues in a broader global environment. By contrast, the walls of the enclosure provide the only global cues available and thus may serve as both global and local cues. Therefore, in the present research we aimed to determine whether the differences in encoding strategies seen with landmark arrays and enclosures reflect the nature of the stimuli (discrete cues vs. extended surfaces) or the nature of the task. Accordingly, we compared the strategies used by pigeons with landmarks (landmark group) and the strategies used by pigeons with a set of walls that did not block external cues (wall group). If pigeons encode information differently from extended surfaces than from discrete landmarks, then the two groups should differ on expansion tests. Specifically, on the basis of the previous studies, we would expect pigeons in the landmark group to show an absolute strategy and pigeons in the wall group to show a mixture of absolute and relational searching. Alternatively, if the availability of external cues is the critical factor, then a difference in strategy among groups should not emerge because the same external cues were available for both groups.

A second purpose of our research was to determine whether both distance and direction are encoded in terms of absolute or relational information or whether these aspects of spatial localization differ in terms of encoding strategy preference. Some evidence suggests that distance and direction from a landmark may be encoded independently. For example, Cheng (1994) trained pigeons to find food at a certain distance from a landmark with a stripe. During training, both the stripe and room cues indicated the direction of the goal from the landmark. On tests, Cheng rotated the landmark, putting the two directional cues in conflict. The pigeons averaged the direction indicated by the competing cues, but they maintained a constant distance from the landmark, which indicated that distance and direction were independent.

Clark's nutcrackers also appeared to determine distance and direction independently. After training nutcrackers to use relational distances from two distinct landmarks to find a goal, Kamil and Jones (2000) tested the birds with rotated arrays. With a $90^{\circ}$ rotation, the birds appeared to determine direction on the basis of absolute bearings rather than on the landmark array: They searched directly north or south of one of the landmarks instead of searching inside the configuration. This result suggests that the nutcrackers encoded the distance and direction separately: Although they learned to use the relationship between the landmarks to determine distance, on the $90^{\circ}$ rotation tests, they did not use the relationship between the landmarks to determine the direction from the landmarks to search.

Gerbils, in contrast, have been shown to use the relationship between landmarks to determine the direction from landmarks to the goal (Collett, Cartwright, \& Smith, 1986). Collett et al. trained gerbils to find the center of three identical landmarks placed in an equilateral triangle. On tests in which the array was rotated $60^{\circ}$, the gerbils searched primarily in the center of the rotated array; however, they showed secondary search peaks at locations defined by absolute bearings. Thus, the gerbils appeared to encode both the relational and the absolute direction from the landmarks to the goal, but the relational bearings seemed to be dominant.

In the present study, we conducted rotation tests to assess how pigeons determine direction from the landmarks or walls. Specifically, would the pigeons determine direction on the basis of absolute bearings, or would the pigeons in either group use relational bearings on the basis of the configuration of the landmarks or walls to determine the direction from these cues to search? If they use relational bearings, they should continue to search in the center even when the array or wall set is rotated. If they use bearings provided by external room cues or internal compass cues, then their search would be deflected away from the center and they should search at the learned absolute direction from a particular landmark or corner when the array or wall set is rotated.

\section{Method}

\section{Subjects}

Twelve adult silver king pigeons (Columbia livia) served as subjects; 6 were trained with the landmark array, and 6 were trained with the wall set. None of the birds had previous experience in the open field or with spatial tasks. The birds were kept at approximately $85 \%$ of their free-feeding weight by feedings of maple peas obtained during experimental sessions and supplemental feedings of Mazuri pigeon pellets. The pigeons were housed in large individual cages on a 12-hr light-dark schedule (lights on at 6 a.m.).

\section{Apparatus}

The testing room was $300 \mathrm{~cm} \times 330 \mathrm{~cm}$ with distinct features on each wall, including the door and covered windows. A $5 \mathrm{~cm}$-high, $200 \mathrm{~cm} \times 200$ $\mathrm{cm}$ wooden tray lined with approximately $3 \mathrm{~cm}$ of aspen chips was centered flush against the south wall, opposite the door. A $7 \times 7$ grid was marked on the floor of the tray with Velcro pieces set $25 \mathrm{~cm}$ apart. We used 
these Velcro pieces to select the location for the landmark array or wall set, and they secured the goal in place. Start and finish boxes were placed flush against the north wall. Strings attached to openings on the boxes ran through a pulley system so that the boxes could be opened and closed from an adjacent observation room. A video camera was mounted on the ceiling above the apparatus.

The landmarks were four identical bottles $(16.5 \mathrm{~cm}$ tall, $7 \mathrm{~cm}$ in diameter) filled with grit. The bottles had black caps, and their sides were covered with silver duct tape and a horizontal strip of white cloth tape. The wall sets were uniform white squares. The initial training wall set was 50 $\mathrm{cm} \times 50 \mathrm{~cm}$ and $9 \mathrm{~cm}$ high. A second training wall set was $50 \mathrm{~cm} \times 50$ $\mathrm{cm}$ and $18 \mathrm{~cm}$ high, and the expansion wall set was $100 \mathrm{~cm} \times 100 \mathrm{~cm}$ and $18 \mathrm{~cm}$ high. A white rectangular goal dish $(10 \mathrm{~cm} \times 5 \mathrm{~cm} \times 2 \mathrm{~cm}$ container with a $2 \mathrm{~cm} \times 2 \mathrm{~cm}$ hole) held maple peas as a reward.

\section{Procedure}

The birds initially were trained in their home cages until they reliably ate from the experimental goal dish and searched for food when it was covered by aspen chips. They were then shaped in the open-field room to enter and exit the start and finish boxes. Pigeons received between four and eight shaping trials per day, depending on their performance.

Next, the birds began training with the goal container centered in either the landmark array or the 9-cm-high wall set. On initial trials, the goal dish was completely uncovered. For the birds trained with the wall set, the wall set initially was pushed as deep as possible into the aspen chips. When the bird quickly and consistently climbed over the edge, the wall set was pulled up gradually over trials so that it eventually rested on top of the aspen chips. This procedure was then repeated with the $18-\mathrm{cm}$ wall set. For the landmark birds, the landmarks were placed in a $50-\mathrm{cm}$ square array. For both groups, the goal dish was gradually buried under the aspen chips across trials, and the number of trials was increased to 10 per day. The goal location varied across trials and was chosen at random from the middle 25 locations marked with Velcro pieces, but the configuration between the landmarks or walls and the goal remained constant on all training trials. The landmark array and wall sets were always aligned according to the main axes of the room on all training trials (i.e., they were translated in the room but not rotated during training). Training continued until the bird located the food within $30 \mathrm{~s}$ of its first search on all 10 trials for 2 consecutive days.

Once the bird reached criterion, we randomly intermixed two nonreinforced trials with eight regular training trials each day to ensure that the birds would continue to search when the goal dish was absent. If the bird did not consistently make 30 searches on the nonreinforced trials, it was trained with a smaller goal dish (a plastic lid $2.5 \mathrm{~cm}$ in diameter). When the bird repeated the previous criteria on the eight reinforced trials and made at least 30 searches on the nonreinforced trials, one test trial and one control replaced the nonreinforced trials. We continued the testing for 15 days to obtain five of each test type and 15 control tests.

Three types of test trials were administered: small rotation tests, large rotation tests, and expansion tests. In addition, control trials that were identical to the nonreinforced training trials were administered. For the small and large rotation tests, the distance between the landmarks or walls remained constant at $50 \mathrm{~cm}$, but the array or wall set was rotated by $15^{\circ}$ or $45^{\circ}$, respectively. The direction of rotation was counterbalanced across birds. During expansion tests, the alignment of the array in the room remained as in training, but the distance between the landmarks or walls was doubled (i.e., $100 \mathrm{~cm}$ apart). Only one test type was administered per day, and the order of tests was counterbalanced across birds to include all possible orders.

\section{Data Analysis}

All test trials and control trials were videotaped and were played on a TV screen so that the location of the first 30 search responses could be marked on a transparent sheet of paper. We defined search response as movement of the beak that resulted in visible displacement of aspen chips. We then determined search coordinates by taping the transparency sheet to the computer screen and using the computer mouse to click on each recorded search location. The computer program recorded the coordinates of each search response in reference to the location of the array.

To determine interrater reliability, the main observer and one independent observer recorded the same trial for one of each type of test for each bird. We then analyzed the search pattern for these tests by determining the peak search location for each set of observations separately and comparing the peak search locations for each trial of the same type through bidimensional regression (Friedman \& Kohler, 2003).

We calculated the peak place of searching for each type of trial for each bird using the iterated median procedure described by Cheng (1989). The median for each axis is calculated and then recalculated over the range on which it centered. For example, if the first median is calculated over the range of $-5 \mathrm{~cm}$ to $30 \mathrm{~cm}$ and the median is $20 \mathrm{~cm}$, the next median would be calculated over the range of $10 \mathrm{~cm}$ to $30 \mathrm{~cm}$. This procedure is repeated until the iterated medians do not change by more than $0.05 \mathrm{~cm}$. This procedure is designed to determine the middle of the highest region in a distribution and is preferred over measures such as the mean or median, which may skew the value toward the center because of outlying searches. Additionally, the spread of searching (calculated by the interquartile range) on control and rotation tests was determined from the final range in the iteration procedure.

In addition, we used the coordinates of each search to calculate the mean proportion of searches in various locations within the array. First, the search space within the array was divided into a grid, with each cell representing a $10 \mathrm{~cm} \times 10 \mathrm{~cm}$ area. We calculated and plotted the mean proportion of searches in each grid area using the procedure described in Gray et al. (2004) to provide a graphical overview of the distribution of search pecks for each trial type. Second, for statistical purposes, we determined the proportion of pecks that fell within a center region (all trial types) or within regions that corresponded to the absolute learned distance from the landmarks or corners of the wall set (expansion tests only). Each of these regions constituted $6.25 \%$ of the total area within the array. Thus, on tests in the small array (i.e., control tests and rotation tests), the center region was a $12.5 \mathrm{~cm} \times 12.5 \mathrm{~cm}$ area, whereas on expansion tests, the center and absolute regions were $25 \mathrm{~cm} \times 25 \mathrm{~cm}$.

The peak search location (iterated median) for each bird and the proportion of searches in each region were calculated from the total searches made across all five tests of each type. The distance of the peak search location from the center, the spread, and the proportion of searches were compared across groups and trial types through analyses of variance (ANOVAs). Furthermore, the proportion of searching in the center and absolute regions were compared with chance levels using chi-square tests. The alpha level was set at .05 except as otherwise noted.

\section{Results}

The interrater reliabilty was $99 \%$ for the control tests; $98 \%$ and $96 \%$ for the small and large rotation tests, respectively; and $99 \%$ for the expansion tests.

\section{Control Tests}

Both groups of pigeons learned the task readily and concentrated their searching in the center of the array or wall set. The calculated peak search locations on the control tests were near the center for all birds (see Table 1). The distribution of searches in the array is depicted at the top right of Figure 1, and the proportion of searches that fell within the center region is shown in Table 1. Chi-square tests revealed that all birds searched significantly more in the center than would be expected by chance $(p s<.0125)$. 
Table 1

Iterated Medians and Proportion of Searches in the Center Region (and Absolute Regions for Expansion Tests) for Each Type of Test Trial for Each Bird

\begin{tabular}{|c|c|c|c|c|c|c|c|c|c|c|c|c|c|}
\hline \multirow[b]{2}{*}{$\begin{array}{l}\text { Bird } \\
\text { ID }\end{array}$} & \multicolumn{3}{|c|}{ Control } & \multicolumn{3}{|c|}{ Small rotation } & \multicolumn{3}{|c|}{ Large rotation } & \multicolumn{4}{|c|}{ Expansion } \\
\hline & $\begin{array}{c}X \text { peak } \\
(\mathrm{cm})\end{array}$ & $\begin{array}{c}\text { Y peak } \\
(\mathrm{cm})\end{array}$ & Prop. & $\begin{array}{c}X \text { peak } \\
(\mathrm{cm})\end{array}$ & $\begin{array}{c}\text { Y peak } \\
(\mathrm{cm})\end{array}$ & Prop. & $\begin{array}{c}\text { X peak } \\
(\mathrm{cm})\end{array}$ & $\begin{array}{c}\text { Y peak } \\
(\mathrm{cm})\end{array}$ & Prop. & $\begin{array}{c}\text { X peak } \\
(\mathrm{cm})\end{array}$ & $\begin{array}{c}\text { Y peak } \\
(\mathrm{cm})\end{array}$ & $\begin{array}{c}\text { Prop. } \\
\text { (Center) }\end{array}$ & $\begin{array}{l}\text { Prop. } \\
\text { (Abs.) }\end{array}$ \\
\hline \multicolumn{14}{|c|}{ Landmark group } \\
\hline 1 & 24.7 & 25.6 & .378 & 23.0 & 29.3 & .413 & 25.3 & 28.8 & .380 & 65.9 & 59.7 & .080 & .387 \\
\hline 2 & 26.1 & 24.7 & .296 & 27.6 & 27.4 & .207 & 24.7 & 23.3 & .266 & 104.7 & 26.7 & .000 & .213 \\
\hline 3 & 26.2 & 24.2 & .389 & 26.0 & 22.1 & .373 & 23.3 & 26.4 & .300 & 67.4 & 56.8 & .000 & .513 \\
\hline 4 & 27.0 & 24.3 & .091 & 22.8 & 21.9 & .207 & 27.5 & 29.8 & .133 & 36.6 & 41.7 & .000 & .267 \\
\hline 5 & 25.3 & 23.4 & .247 & 24.8 & 21.9 & .267 & 26.2 & 22.8 & .227 & 37.5 & 74.9 & .013 & .387 \\
\hline 6 & 23.6 & 26.7 & .200 & 27.5 & 24.0 & .227 & 23.2 & 27.8 & .280 & 66.5 & 62.4 & .040 & .227 \\
\hline Overall & 25.5 & 24.8 & .267 & 25.3 & 24.4 & .282 & 25.0 & 26.5 & .264 & 63.1 & 53.7 & .022 & .332 \\
\hline \multicolumn{14}{|c|}{ Wall group } \\
\hline 7 & 24.9 & 24.2 & .527 & 25.1 & 23.1 & .565 & 25.2 & 23.4 & .592 & 71.2 & 66.2 & .000 & .697 \\
\hline 8 & 23.7 & 22.4 & .525 & 23.0 & 23.7 & .433 & 25.4 & 22.6 & .466 & 28.1 & 25.8 & .040 & .673 \\
\hline 9 & 24.8 & 22.8 & .393 & 24.9 & 20.7 & .380 & 24.7 & 28.4 & .447 & 24.6 & 40.9 & .007 & .493 \\
\hline 10 & 26.2 & 22.0 & .456 & 24.2 & 19.7 & .333 & 25.0 & 19.9 & .373 & 32.9 & 74.8 & .007 & .667 \\
\hline 11 & 26.8 & 22.9 & .539 & 26.1 & 22.6 & .387 & 24.8 & 24.2 & .467 & 29.3 & 23.0 & .047 & .473 \\
\hline 12 & 24.2 & 23.4 & .492 & 25.9 & 20.8 & .241 & 22.7 & 21.2 & .433 & 22.5 & 34.5 & .000 & .470 \\
\hline Overall & 25.1 & 23.0 & .489 & 24.9 & 21.8 & .390 & 24.6 & 23.3 & .463 & 34.8 & 44.2 & .017 & .579 \\
\hline
\end{tabular}

Note. X peak represents the distance from the line connecting the two leftmost (eastern) landmarks or corners, and Y peak represents the distance from the line connecting the two bottom (north) landmarks or corners. The center region represents $6.25 \%$ of the area in the array. The four absolute regions (expansion only) total $25 \%$ of the area in the array. The center of the array or wall set is $25 \mathrm{~cm}$ for the control, small rotation, and large rotation tests and is $50 \mathrm{~cm}$ for the expansion tests. Prop. = proportion; abs. = absolute.

\section{Expansion Tests}

On the expansion tests, neither group of pigeons concentrated their searches primarily in the center. Upon examination of the iterated medians (see Table 1), we observed that the peak search place on the expansion tests was closer to the absolute learned distance from the corners than to the center for all subjects in the wall group. For 4 of the birds in the landmark group (Birds 1, 3, 4, and 6), the peak search place was in between the absolute learned distance and the center; for the remaining 2 landmark birds, 1 showed a peak search area closer to the absolute learned distance (Bird 5) and 1 showed a peak search area outside the landmark array (Bird 2). Figure 1 shows the distribution of searching within the array. We conducted an ANOVA to compare the proportion of searches in the center region with the proportion of searches in the same-sized regions defined by the absolute distance. Because there are four absolute regions, and hence 4 times the area in which a bird using an absolute strategy could search, we divided the proportion of searches in the absolute distance area by 4 to control for opportunity. The ANOVA revealed a significant Group $\times$ Search Area interaction, $F(1,10)=6.18, p=.03$. Further comparison revealed that both groups of birds searched in the corner areas significantly more than they did in the center area: landmark group, $F(1,5)=6.6, p=.05$; wall group, $F(1,5)=$ $12.26, p=.02$. The number of searches in the center region did not differ between groups, $F(1,10)<1, p=.73$; however, the wall group searched significantly more in the corner regions than did the landmark group, $F(1,10)=7.55, p=.02$.

Finally, the proportion of searches in the center and absolute regions were compared with the level expected by chance. Chi- square tests revealed that 4 of the birds trained with landmarks and 4 of the birds trained with walls searched in the center significantly less than chance $(p s<.02)$. The remaining birds in each group showed chance levels of searching in the center region; no birds responded higher than chance levels in the center region. Three of the birds trained with landmarks and all 6 birds trained with walls showed greater-than-chance-level searching in the absolute regions $(p s<.001)$. No birds searched at less than chance level in the absolute regions.

\section{Small and Large Rotation Tests}

Because data from the control and rotation tests were entered into several analyses, the alpha level was set at .0125 . On small and large rotation tests, both groups of pigeons searched primarily in the center (see Figure 1). The peak search locations (see Table 1) for both the small and large rotation tests were near the center. We conducted an ANOVA to compare the distance from peak to center across control and rotation tests and across groups. No significant differences were seen across trial types or groups ( $p \mathrm{~s}>$ .05). Of interest, a significant difference was seen between the $x$ and $y$ dimensions, in which the peak was farther from the center on the vertical axis, $F(1,10)=25.41, p=.001$.

The proportion of searches in the center region was compared across control and rotation tests and across groups. The birds trained with walls searched in the center significantly more than did the birds trained with the landmark array, $F(1,10)=14.44$, $p=.003$. No significant differences were seen across trial types $(p>.05)$, and the Trial Type $\times$ Condition interaction just failed to reach significance, $p=.03$. Additionally, a chi-square test 


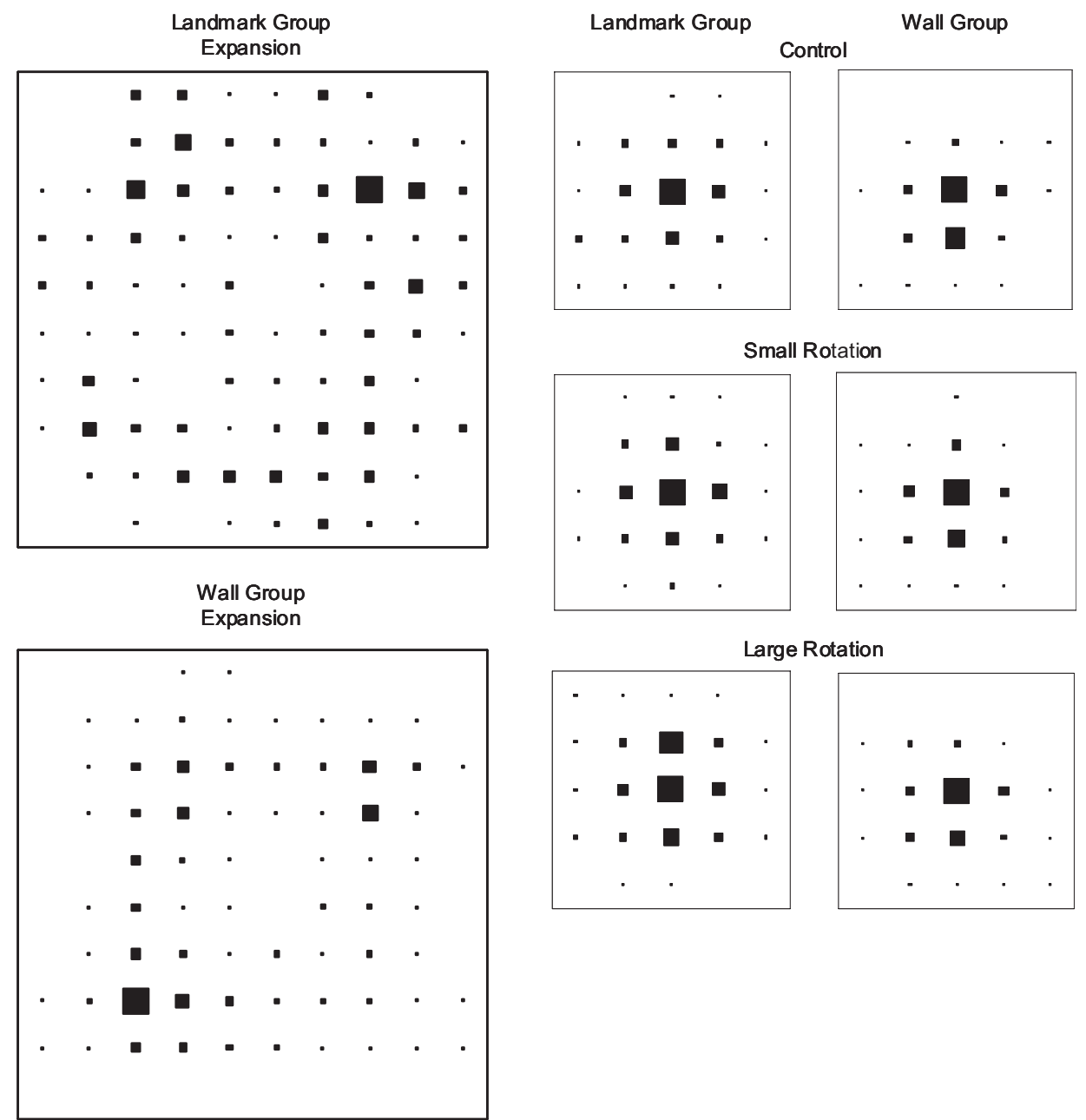

Figure 1. Proportion of searches in $10 \mathrm{~cm} \times 10 \mathrm{~cm}$ grid sections of the search area within the perimeter of the landmark array or wall set on each type of test. Larger squares indicate a higher proportion of searching behavior in that location.

revealed that all birds had a higher proportion of searches in the center region than would be expected by chance $(p s<.01)$.

Finally, we used an ANOVA to compare the spread of searches across trial types and groups. No significant differences were found for group type or trial type or between the horizontal and vertical dimensions $(p \mathrm{~s}>.05)$. The wall group had a significantly smaller search spread than did the landmark group, $F(1,10)=$ 12.22, $p=.006$.

\section{Discussion}

Pigeons in both the landmark group and the wall group learned to locate the center of the training apparatus. On the expansion tests, pigeons searched at the absolute distance from a landmark or corner, regardless of whether they were trained with landmarks or walls. Thus, it seems that the previously observed strategy differences are not due to a difference in encoding strategies for landmarks and extended surfaces (Gray et al., 2004; Spetch et al., 1997). Rather, our results suggest that when external cues are not blocked, pigeons tend to use an absolute distance strategy regard- less of whether they are encoding information from landmarks or from walls.

The difference between the peak search locations of the pigeons in our wall group and the peak search locations of the pigeons tested by Gray et al. (2004) suggests that the availability of external cues may be an important determinant of how spatial information is encoded. One interesting possibility is that the presence of external cues alters the set of cues that constitute global information. In the present study, because the external cues were not blocked, the shape and features of the larger experimental room would make up the set of global cues, and the array of walls or landmarks would serve as local cues to indicate the goal location. In contrast, when external cues are blocked, the walls of the experimental apparatus itself may provide the only global information.

A difference in encoding global information and encoding local information is supported by studies in which researchers examined brain lateralization. In particular, studies have shown that the right hemisphere of the avian brain is concerned primarily with global 
aspects of the environment, whereas the left hemisphere is concerned with local cues (Kahn \& Bingman, 2004; Tommasi, Gagliardo, Andrew, \& Vallortigara, 2003; Tommasi \& Vallortigara, 2001). For example, chicks tested with the left eye were more likely to search according to the global shape of the room, whereas chicks tested with the right eye were more likely to use the landmark or absolute learned distances when determining a search location (Tommasi \& Vallortigara, 2001). Similarly, pigeons and chicks with intact hippocampal formations or lesions to the left hippocampal formation have been shown to rely on the global room cues to search for the reward, whereas pigeons with lesions to the right hippocampal formation tend to rely more on local landmark cues (Kahn \& Bingman, 2004; Tommasi et al., 2003).

Results of the current study are consistent with the theory that local and global cues are encoded and used differently. In particular, our results showed that when either landmarks or walls serve as local cues in a larger global environment, pigeons tend to rely on absolute, rather than relational, distance.

Interestingly, although pigeons in both groups searched according to absolute distances on expansion tests, the results of the rotation tests suggest that they learned the direction to the goal in relation to the whole array. In particular, no differences were observed between the control and rotation tests in terms of the proportion of searches in the center or the spread of searching, indicating that pigeons did not follow an absolute direction from one landmark. These results contrast with those of Kamil and Jones $(1997,2000)$, in which it was shown that nutcrackers rely predominantly on absolute bearings.

The discrepancy between our results and those of Kamil and Jones $(1997,2000)$ could reflect a species difference, but this seems unlikely because nutcrackers and pigeons have shown qualitatively similar responses on landmark expansion tests. Another, perhaps more likely, alternative is that the difference reflects the number and/or distinctiveness of the landmarks used. On rotation tests with the two distinct landmarks, the nutcrackers chose a search location that was directionally correct according to one landmark but directionally incorrect according to the other landmark. The chosen search location resulted in the incorrect landmark being more distal to the search location than the correct landmark, and hence the birds may have attended to it less while searching. This finding is comparable to the gerbils trained in the equilateral triangle array (Collett et al., 1986). In that case, when the gerbils did not search according to the relational direction between landmarks, they searched at a location that was directionally correct according to two of the landmarks. Specifically, the rotated triangle array produces three locations outside the array in which absolute learned directions from the closest two landmarks converge on a single location (Figure 2). This location is directionally incorrect according to the third, but most distal, landmark.

In contrast, when the four-landmark array is rotated, there are no locations at which absolute learned vectors will converge (see Figure 2). Thus, if searching according to absolute learned directions, the birds must rely on only one landmark at a time. Moreover, in many of the search locations defined by absolute vectors, at least one of the directionally incorrect landmarks or walls would be closer to the chosen search location than the reference landmark or wall. Given the evidence that pigeons treat the array as a unit or configuration for the purpose of identifying landmarks (Spetch et al., 1997), it is unlikely that they would choose a search location
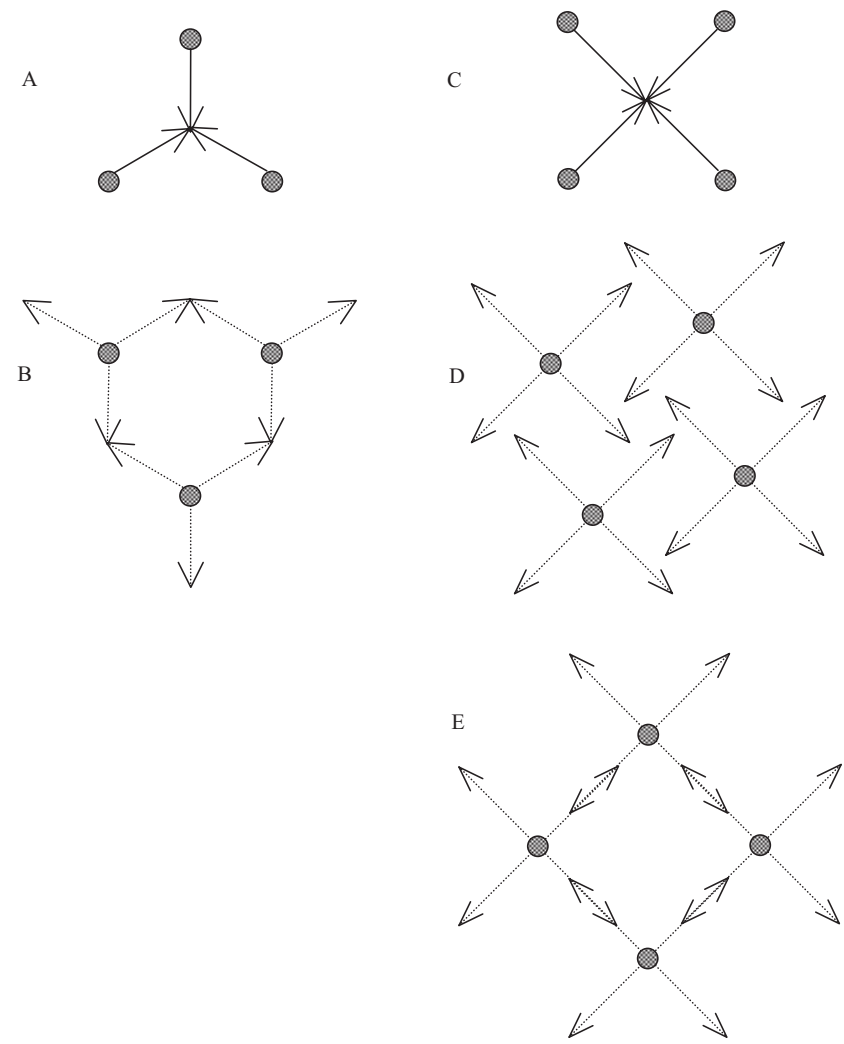

Figure 2. Schematic representation of directional vectors for equilateral triangle and square arrays. A: Vectors learned during training sessions on triangle array (gerbils: Collett, Cartwright, \& Smith, 1986). B: Absolute direction patterns when the array is rotated by $60^{\circ}$. C: Vectors learned during training on a square array. D and E: Absolute direction patterns when the array is rotated by $15^{\circ}$ and $45^{\circ}$.

that is far outside the array. Consequently, the birds may have resisted searching at locations dictated by absolute bearings because this would lead to searching either far outside the array or at a location that is directionally incorrect from a landmark that is closer than the referent one. Future researchers need to determine whether these speculations have merit. It should also be noted that although our results suggested relational bearings, whereas those by Kamil and Jones (1997, 2000) suggested absolute bearings, the two sets of results are consistent in providing support for the theory that distance and direction are encoded separately.

Although pigeons in the landmark and wall groups showed similar strategies on expansion and rotation tests, some systematic differences were observed in their search behavior. The birds trained with the walls showed more focused searching in the center on control and rotation tests and more searching in the absolute regions on expansion tests than did the birds trained with landmarks. One possible reason for this difference is that the pigeons in the wall group had to perform the salient action of climbing over the wall set before searching for the goal. Although pigeons trained in the landmark group had to enter the array, the required action may be far less salient because it involves only visual processing as opposed to processing both visual and kinesthetic information Alternatively, the pigeons trained with wall sets might have been 
more accurate in their search simply because they had more information with which to work. That is, the birds in the wall condition localized the goal on the basis of solid walls, which provide more surface area than do the landmarks alone.

Despite the differences in precision of searching with walls and landmarks, the strategies of cue use were similar whether the birds were searching according to walls or according to discrete landmarks. In both cases, expansion tests indicated that the birds encoded absolute distance from landmarks or walls, and rotation tests suggested that the direction of search from the landmarks or walls was encoded in relation to the array. Our results contribute to the understanding of spatial cognition in three ways. First, they indicate that the previously observed differences between searching in enclosures versus searching in landmark arrays does not reflect a difference between extended surfaces and discrete landmarks but, instead, likely reflects the availability of external directional cues. These external cues may alter the encoding of global and local information. Second, our results provide additional evidence that pigeons learn about the array of cues: In addition to using the array for a landmark matching process (Cheng \& Spetch, 1998), they also use the array to determine the direction to search. Finally, our results provide further support for Cheng's (1994) conclusion that distance and direction are encoded by pigeons as independent sources of spatial information rather than as a unified vector.

\section{References}

Cheng, K. (1986). A purely geometric module in the rat's spatial representation. Cognition, 23, 149-178.

Cheng, K. (1989). The vector sum model of pigeon landmark use. Journal of Experimental Psychology: Animal Behavior Processes, 15, 366-375.

Cheng, K. (1994). The determination of direction in landmark-based spatial search in pigeons: A further test of the vector sum model. Animal Learning \& Behavior, 22, 291-301.

Cheng, K., \& Newcombe, N. S. (2005). Is there a geometric module for spatial orientation? Squaring theory and evidence. Psychonomic Bulletin \& Review, 12, 1-23.

Cheng, K., \& Spetch, M. L. (1998). Mechanisms of landmark use in mammals and birds. In S. Healy (Ed.), Spatial representation in animals (pp. 1-17). Oxford, England: Oxford University Press.

Collett, T. S., Cartwright, B. A., \& Smith, B. A. (1986). Landmark learning and visuo-spatial memories in gerbils. Journal of Comparative Physiology A, 158, 835-851.

Friedman, A., \& Kohler, B. (2003). Bidimensional regression: Assessing the configural similarity and accuracy of cognitive maps and other two-dimensional data sets. Psychological Methods, 8, 468-491.

Gallistel, C. K. (1990). The organization of learning. Cambridge, MA: MIT Press.

Gray, E. R., Spetch, M. L., Kelly, D. M., \& Nguyen, A. (2004). Searching in the center: Pigeons encode relative distances from walls of an enclosure. Journal of Comparative Psychology, 118, 113-117.

Jones, J. E., Antoniadis, E., Shettleworth, S. J., \& Kamil, A. C. (2002). A comparative study of geometric rule learning by nutcrackers (Nucifraga columbiana), pigeons (Columba livia), and jackdaws (Corvus monedula). Journal of Comparative Psychology, 116, 350-356.
Kahn, M. C., \& Bingman, V. P. (2004). Lateralization of spatial learning in the avian hippocampal formation. Behavioral Neuroscience, 118, 333-344.

Kamil, A. C., \& Jones, J. E. (1997, November 20). The seed-storing corvid Clark's nutcracker learns geometric relationships among landmarks. Nature, 390, 276-279.

Kamil, A. C., \& Jones, J. E. (2000). Geometric rule learning by Clark's nutcrackers (Nucifraga columbiana). Journal of Experimental Psychology: Animal Behavior Processes, 26, 439-453.

Kelly, D. M., \& Spetch, M. L. (2001). Pigeons encode relative geometry. Journal of Experimental Psychology: Animal Behavior Processes, 27, 417-422.

Kelly, D. M., Spetch, M. L., \& Heth, D. C. (1998). Pigeons' (Columbia livia) encoding of geometric and featural properties of a spatial environment. Journal of Comparative Psychology, 112, 259-269.

Learmonth, A. E., Nadel, L., \& Newcombe, N. S. (2001). Children's use of landmarks: Implication for modularity theory. Psychological Science, $13,337-341$.

Sovrano, V. A., Bisazza, A., \& Vallortigara, G. (2005). Animals' use of landmarks and metric information to reorient: Effects of the size of the experimental space. Cognition, 97, 121-133.

Spetch, M. L., Cheng, K., MacDonald, S. E., Linkenhoker, B. A., Kelly, D. M., \& Doerkson, S. R. (1997). Use of landmark configuration in pigeons and humans: II. Generality across search tasks. Journal of Comparative Psychology, 111, 14-24.

Spetch, M. L., \& Kelly, D. M. (2006). Comparative social cognition: Processes in landmark and surface-based place finding. In E. A. Wasserman \& T. R. Zentall (Eds.), Comparative cognition experimental explorations of animal intelligence (pp. 210-228). Oxford, England: Oxford University Press.

Spetch, M. L., Rust, T. B., Kamil, A. C., \& Jones, J. E. (2003). Searching by rules: Pigeons' landmark-based search according to constant bearing or constant distance. Journal of Comparative Psychology, 117, 125-132.

Tommasi, L., Gagliardo, A., Andrew, R. J., \& Vallortigara, G. (2003). Separate processing mechanisms for encoding of geometric and landmark information in the avian hippocampus. European Journal of Neuroscience, 17, 1695-1702.

Tommasi, L., \& Vallortigara, G. (2000). Searching for the center: Spatial cognition in the domestic chick (Gallus gallus). Journal of Experimental Psychology: Animal Behavior Processes, 26, 477-486.

Tommasi, L., \& Vallortigara, G. (2001). Encoding of geometric and landmark information in the left and right hemispheres of the avian brain. Behavioral Neuroscience, 115, 602-613.

Tommasi, L., Vallortigara, G., \& Zanforlin, M. (1997). Young chickens learn to localize the center of a spatial environment. Journal of Comparative Physiology A, 180, 248-254.

Vallortigara, G., Feruglio, M., \& Sovrano, V. A. (2005). Reorientation by geometric and landmark information in environments of different size. Developmental Science, 8, 393-401.

Vallortigara, G., Zanforlin, M., \& Pasti, G. (1990). Geometric modules in animal's spatial representation: A test with chicks. Journal of Comparative Psychology, 104, 248-254.

Received March 10, 2006

Revision received June 14, 2006 Accepted June 20, 2006 